\title{
EVALUACIÓN DEL RIESGO CARDIOVASCULAR EN ADULTOS DEL DISTRITO MUNICIPAL DE SANTANA, PERAVIA, REPÚBLICA DOMINICANA, DURANTE EL PERIODO NOVIEMBRE - DICIEMBRE 2019 (ERCAS I)
}

\section{Cardiovascular risk evaluation in adults of the municipal district of Santana, Peravia, Dominican Republic during the November-December 2019 period (CREASI)}

\author{
Ashley Karina Reyes Guzmána , Karla Yorián Martínez Mercadob, \\ Michelle Marie Jiménez Mieses ${ }^{c}$ y Ramón Antonio Romano Peraltad
}

Recibido: 12 de febrero, $2020 \bullet$ Aprobado: 6 de mayo, 2020

Cómo citar: Reyes Guzmán AK, Martínez Mercado KY, Jiménez Mieses MM, Romano Peralta RA. Evaluación del riesgo cardiovascular en adultos del distrito municipal de Santana, Peravia, República Dominicana, durante el periodo noviembre - diciembre 2019 (ERCAS I). cysa [Internet]. 15 de febrero de 2021 [citado 23 de febrero de 2021];5(1):97-107. Disponible en: https://revistas.intec. edu.do/index.php/cisa/article/view/2057

\section{Resumen}

Introducción: las enfermedades cardiovasculares comprenden un conjunto de trastornos del corazón y de los vasos sanguíneos, actualmente ocupan el primer lugar en causa de muertes a nivel mundial. Las enfermedades cardiovasculares pueden ser prevenidas mediante la estimación del riesgo cardiovascular del paciente y abordaje de su factor de riesgo cardiovascular.

Objetivo general: evaluar el riesgo cardiovascular en adultos del distrito municipal de Santana, Peravia, República Dominicana, durante el periodo noviembre - diciembre 2019.

Metodología: estudio de observacional, prospectivo, de corte transversal y analítico en el cual se analizaron 160 pacientes para evaluar el riesgo cardiovascular y factores de

\footnotetext{
a Interno de medicina del Instituto Tecnológico de Santo Domingo (INTEC), Sanot Domingo, República Dominicana.

ORCID: 0000-0002-7661-6181

Correo-e: 1061711@est.intec.edu.do

b Interno de medicina del Instituto Tecnológico de Santo Domingo (INTEC), Sanot Domingo, República Dominicana.

ORCID: 0000-0002-3720-5523,

Correo-e: 1056160@est.intec.edu.do
}

\begin{abstract}
Introduction: Cardiovascular diseases include a set of disorders of the heart and blood vessels that currently occupy the main cause of deaths worldwide. Cardiovascular diseases can be prevented by estimating the patient's cardiovascular risk and addressing their cardiovascular risk factor.

General objective: To assess cardiovascular risk in adults in the municipal district of Santana, Peravia, Dominican Republic during the period November - December 2019.

Methods: observational, cross-sectional, prospective and analytical study in which 160 patients were analyzed to assess the cardiovascular risk and associated risk factors in the Santana municipal district with the American College of Cardiology (ACC) risk estimator.
\end{abstract}

\footnotetext{
${ }^{c}$ Interno de medicina del Instituto Tecnológico de Santo Domingo (INTEC), Sanot Domingo, República Dominicana. ORCID: 0000-0002-1404-0826

Correo-e: 1065436@est.intec.edu.do

d Interno de medicina del Instituto Tecnológico de Santo Domingo (INTEC), Sanot Domingo, República Dominicana.

ORCID: 0000-0002-3659-6665

Correo-e:1067015@est.intec.edu.do
} 
riesgo asociados con el estimador de riesgo del American College of Cardiology (ACC).

Resultados: el $36.26 \%$ de la población estuvo en riesgo cardiovascular bajo a 10 años; la media de riesgo cardiovascular de por vida fue $47.7 \%$ en el sexo masculino y $35.5 \%$ en el sexo femenino; los factores de riesgo cardiovascular de dislipidemia (con un $56.3 \%$ ), hiperglicemia (con un 65.6 \%) e hipertensión arterial (HTA) (33.1\%) fueron los más frecuentes. La correlación entre síndrome metabólico y riesgo cardiovascular fue negativa $\left(\mathrm{R}^{2}=0.037\right)$.

Conclusiones: el riesgo cardiovascular a 10 años de la población fue predominantemente bajo, con una minoría de la población teniendo altos niveles. Esto coincide con una alta frecuencia de factores de riesgo cardiovasculares modificables. No se encontró relación entre los criterios diagnósticos de síndrome metabólico y riesgo cardiovascular. Los principales factores hallados fueron dislipidemia, hiperglicemia y HTA.

Recomendaciones: la Organización Mundial de la Salud (OMS) y la American Heart Association (AHA), recomiendan la prevención primaria y la educación sobre los factores de riesgo como las mejores medidas a tomar para disminuir la alta prevalencia de enfermedades cardiovasculares.

Palabras clave: medicina Interna; cardiología; enfermedad cardiovascular; factor de riesgo; hipertensión arterial; diabetes mellitus.

\section{Introducción}

Las enfermedades cardiovasculares (ECV) comprenden un conjunto de trastornos del corazón y de los vasos sanguíneos que se clasifican en hipertensión arterial (HTA), cardiopatía coronaria, enfermedad cerebrovascular, la diabetes mellitus (la cual fue incluida dentro de la clasificación de enfermedades cardiovasculares), entre otras. Las ECV son la primera causa de muerte a nivel mundial según la Organización Mundial de la Salud (OMS); en 2016 representó un $31 \%$ de las muertes totales ${ }^{1}$. La mayoría de estas ECV son prevenibles si se abordan los factores de riesgo y su riesgo cardiovascular ${ }^{2}$. El concepto de riesgo cardiovascular (RCV) hace referencia a la probabilidad de sufrir una enfermedad
Results: $36.26 \%$ of the population had a low 10 -year cardiovascular risk; the mean lifetime cardiovascular risk was $47.7 \%$ in males and $35.5 \%$ in females; cardiovascular risk factors like dyslipidemia (with 56.3\%), hyperglycemia (with 65.6\%) and arterial hypertension (33.1\%) were the most frequent. The correlation between metabolic syndrome and cardiovascular risk was negative $\left(\mathrm{R}^{2}=0.037\right)$.

Conclusions: 10-year cardiovascular risk was predominantly low, with a minority of the population having high levels. This coincides with a high frequency of modifiable cardiovascular risk factors. No correlation was found between the diagnostic criteria for metabolic syndrome and cardiovascular risk. The main cardiovascular risk factors found were dyslipidemia, hyperglycemia and HTA.

Recommendations: The World Health Organization (WHO) and the American Heart Association (AHA) recommend that primary prevention and education on risk factors are the best measures to be taken for the assessment of cardiovascular disease, in order to reduce its prevalence.

Keywords: Internal Medicine; Cardiology; Cardiovascular Diseases; Risk Factors; Arterial Hypertension; Diabetes Mellitus.

cardiovascular en un lapso determinado de tiempo, que habitualmente es entre 5 y 10 años. Todas las personas tienen al menos un porcentaje de riesgo cardiovascular (riesgo cardiovascular óptimo, el mismo estará dado por los factores no modificables del paciente como es la edad, el sexo, la raza y los antecedentes familiares) ${ }^{2,3}$. El RCV sirve como una herramienta clínica para la evaluación cardiovascular del paciente, tomando en cuenta las diferentes variables de este como edad, sexo, raza, niveles de colesterol, hábitos tóxicos, antecedentes personales y familiares. Estos datos se introducen en la calculadora de riesgo, para arrojar la probabilidad de que el paciente padezca un evento cardiovascular en los próximos 10 años (riesgo cardiovascular a 10 años), durante toda su vida (riesgo cardiovascular global, 
probabilidad de presentar un evento en un periodo determinado durante toda la vida del paciente) y el riesgo cardiovascular óptimón

Se estima que, en el 2013, aproximadamente 17.3 millones de personas fallecieron de enfermedades cardiovascular, con $80 \%$ de estas muertes ocurriendo en países con ingresos bajos/medios 5 . El riesgo y las enfermedades cardiovasculares han aumentado su prevalencia e incidencia, principalmente en países en vía de desarrollo como la República Dominicana, evidenciado en el Estudio Factores de Riesgos Cardiovasculary Sindrome Metabólico en la República Dominicana (EFRICARD II).

En el distrito municipal de Santana, según la Dirección Provincial de Salud de Baní, Peravia, hasta el tamizaje de mayo-agosto del 2019, 234 pacientes padecían de hipertensión arterial y 68 de diabetes mellitus. Relacionando esto con la guía de Riesgo Cardiovascular y Enfermedades Cardiovasculares de la American Heart Association (ASCV-AHA), la población de Santana presentaba factores de riesgo para la expresión de un riesgo cardiovascular elevado y padecer de eventos cardiovasculares. Por esta razón, se decidió que el objetivo general de la investigación fuese evaluar el riesgo cardiovascular en adultos del distrito municipal de Santana, Peravia, República Dominicana durante el periodo noviembrediciembre 2019, por sus siglas ERCAS I. Los objetivos específicos fueron: determinar el riesgo cardiovascular a 10 años, óptimo y de por vida de la población de estudio; los factores de riesgo cardiovascular presentes en dicha población; y su distribución por grupo etario y sexo. Por último, realizar un análisis de regresión lineal entre el riesgo cardiovascular a 10 años y el síndrome metabólico.

\section{Materiales y método}

Esta investigación fue observacional, pues se limitó a solo medir las variables sin ejercer control sobre el factor de estudio; de corte transversal ya que las variables fueron medidas en una sola ocasión y no se les dio seguimiento; prospectivo, debido a que la información obtenida es de fuentes primarias, recolectada de forma directa por los investigadores a partir de los sujetos que se estudiaron; analítico, porque se buscó causalidad entre las variables de estudio, con el fin de encontrar una dependencia en la expresión de las mismas estudiadas en los pacientes. La población de estudio constó de 8,393 pacientes los cuales fueron tamizados entre el mes de mayo y agosto del año 2019 por el personal del centro de primer nivel de Santana. En relación con el tamaño de la muestra se utilizó la fórmula de la población finita:

Donde: $n=$ tamaño de la muestra; $N=$ tamaño de la población; $Z$ = valor de $Z$ crítico, el mismo dependió del nivel de confianza que se utilizó el cual fue de $95 \%$, correspondiendo a un $Z_{0.05}$ igual a 1.96; $\sigma^{2}=$ varianza de la población en estudio (que es el cuadrado de la desviación estándar), el mismo se obtuvo de un estudio titulado "Factores de riesgo cardiovascular en la región de Murcia, España” para calcular mediante la fórmula de la población anteriormente mencionada; $d=$ nivel de precisión absoluto o error que se pretendió cometer, en este caso se utilizó un nivel de precisión absoluto de 5 .

Una desviación estándar de 33.8 correspondiente a la variable "Colesterol total". Se utilizó un nivel de confianza de un $95 \%$, para un total de 160 pacientes. Para poder formar parte de la investigación fue necesario que los sujetos de estudio se encontraran entre el rango de edad de 20 a 75 años y residir en el distrito municipal de Santana.

La herramienta de estudio fue un cuestionario realizado por los investigadores y asesores de la investigación, aplicado a los pacientes que asistían al centro de primer nivel de Santana, al igual que aquellos a los cuales se les realizó una visita domiciliaria durante la estadía en la comunidad. Se evaluaron variables tanto categóricas y numéricas como edad, sexo, estado civil, nacionalidad, presión arterial, glucemia, talla, peso, índice de masa corporal (IMC), circunferencia 
abdominal, frecuencia cardíaca, niveles de colesterol total, colesterol HDL, triglicéridos. Para la obtención del colesterol LDL, se utilizaron los valores lipídicos anteriormente mencionados, usando la fórmula de Fridewald, a través del programa en el portal web de Merck Manuals, en su sección de calculadoras médicas. Las pruebas realizadas con relación a los valores lipídicos eran llevadas diariamente desde Santana a Santo Domingo y fueron procesadas en el laboratorio clínico del Hospital Central de Las Fuerzas Armadas (HCFFAA).

Estos valores fueron utilizados para calcular el riesgo cardiovascular a 10 ańos, el riesgo cardiovascular global y óptimo del paciente utilizando el Cardiovascular Risk Estimator del American College of Cardio$\operatorname{logy}$ (ACC), que evalúa la edad, el sexo, la raza, los valores de presión sistólica, diastólica, valores lipídicos anteriormente mencionados, antecedentes personales de diabetes mellitus (DM), hipertensión arterial (HTA), si está en un tratamiento para la hipertensión arterial, si toma aspirina o estatinas. Subsecuentemente, se utilizaron los datos recolectados para realizar el diagnóstico de síndrome metabólico en la población estudiada, usando los criterios establecidos por el American College of Endocrinology (ACE) y el National Health Institute (NIH) de los Estados Unidos. En las gráficas de la investigación no se trabajó con la totalidad de la muestra debido a que no todos los pacientes cumplieron con los requisitos que admite la calculadora de riesgos utilizada para calcular los tres riesgos de estudio, estos incluyen rangos de edad específicos para cada riesgo, niveles de presión arterial, colesterol, entre otros.

Se aplicó previo consentimiento informado donde se describe el objetivo de la investigación y la confiabilidad de los datos. No se expone la identidad del entrevistado respetando su derecho a la privacidad e integridad. Los datos fueron analizados y tabulados mediante el programa Microsoft Excel 2013, programa utilizado, de igual forma, para elaborar el análisis de regresión lineal entre el riesgo cardiovascular a 10 años y el Síndrome Metabólico.

\section{Resultados}

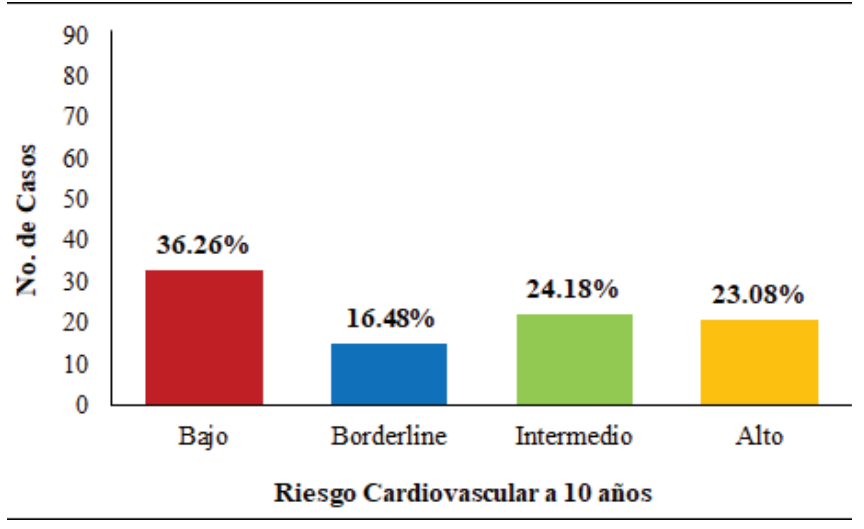

Figura 1. Riesgo cardiovascular a 10 ańos $(\mathrm{n}=91)$

Fuente: elaborado por Jiménez, Martínez, Reyes y Romano, noviembre - diciembre 2019.

En la presente figura se muestra el riesgo cardiovascular a 10 ańos. Se puede observar que un 36.26 $\%$ representa un RCV bajo, correspondiendo a 33 casos. Se estimó que un $16.48 \%$ corresponde a pacientes con un RCV límite, correspondiendo a 15 casos. Por otro lado, un $24.18 \%$ de los pacientes tienen un RCV intermedio, correspondiente a 22 casos. Con respecto al RCV alto, un $23.08 \%$ de los pacientes se encuentran dentro de este rango, correspondiendo a 21 casos.

Tabla 1. Media del riesgo global y riesgo óptimo $(\mathrm{n}=60)$

\begin{tabular}{|c|c|c|}
\hline Sexo & $\begin{array}{c}\text { Media riesgo } \\
\text { cardiovascular de } \\
\text { por vida }\end{array}$ & $\begin{array}{c}\text { Media riesgo } \\
\text { cardiovascular } \\
\text { óptimo }\end{array}$ \\
\hline Masculino & 47.87 & 2.34 \\
\hline Femenino & 35.5 & 1.07 \\
\hline
\end{tabular}

Fuente: elaborado por Jiménez, Martínez, Reyes y Romano, noviembre - diciembre 2019. 
En esta tabla se puede apreciar la media de los valores del riesgo cardiovascular de por vida, y la media de los valores del RCV óptimo, según el sexo. Se evidencia que la media RCV de por vida en el sexo masculino fue de un $47.87 \%$, valor que se encuentra alejado del RCV óptimo, que corresponde a un 2.34 $\%$. Con relación al sexo femenino, se mostró que la media RCV de por vida fue de un $35.5 \%$, mientras que el del riesgo óptimo fue de un $1.07 \%$.

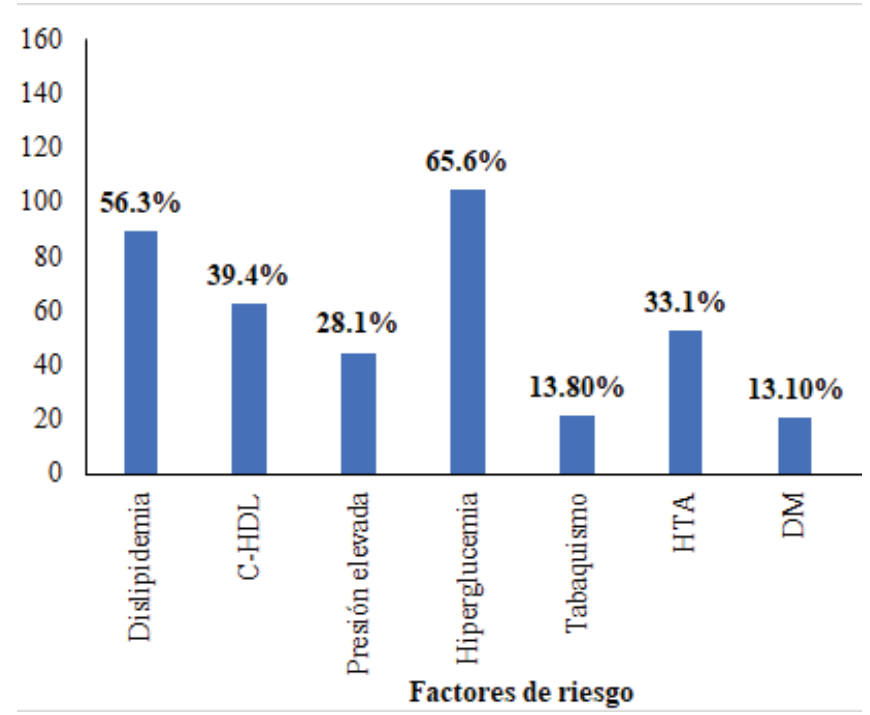

Figura 2. Factores de riesgo cardiovasculares en la población de Santana $(\mathrm{n}=160)$

Fuente: elaborado por Jiménez, Martínez, Reyes y Romano, noviembre - diciembre 2019.

De acuerdo con los resultados obtenidos, se evidenció que un $56.3 \%$ de los pacientes presentó dislipidemia, correspondiendo a 95 casos; en cuanto a C-HDL 39.4 \%, 63 casos, con un nivel por debajo del límite normal; asimismo, $28.1 \%$ presentó elevación de la presión arterial, correspondiendo a 45 casos. En cuanto a hiperglucemia, $65.6 \%$, es decir, 105 casos; respecto a tabaquismo $13.80 \%$, correspondiendo a 22 casos; $33.1 \%$ presentó HTA, esto es, 53 casos. En relación con la DM, un 13.10 \%, 21 casos; y en cuanto a obesidad, $33.1 \%$, lo que refiere a 53 casos.

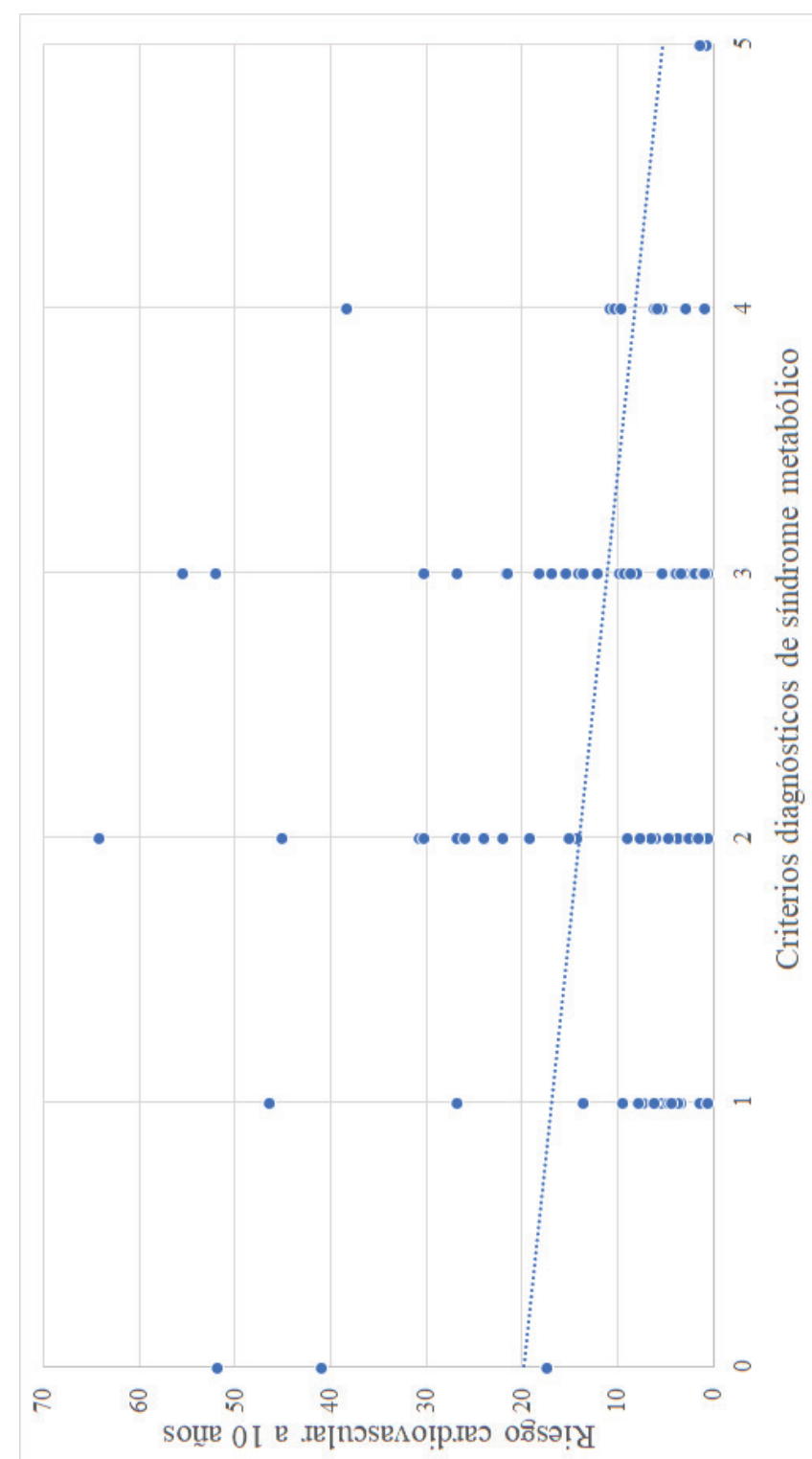

Figura 3. Relación síndrome metabólico y riesgo cardiovascular a 10 años $(n=91)$

Fuente: elaborado por Jiménez, Martínez, Reyes y Romano, noviembre - diciembre 2019.

En la figura 3 se evalúa la relación del síndrome metabólico con el riesgo cardiovascular a 10 años de los pacientes. Se ha encontrado una correlación negativa $\left(\mathrm{R}^{2}=0.037\right)$. Se puede decir que, por cada criterio diagnóstico de síndrome metabólico que tenga el paciente, el riesgo cardiovascular aumenta un 0.037 , lo que demuestra ser poco significativo. 
Tabla 2. Factores de riesgo cardiovascular por sexo y grupo etario $(\mathrm{n}=160)$

\begin{tabular}{|l|c|c|c|c|c|}
\hline \multicolumn{5}{|c}{ Factores cardiovasculares por grupo etario y sexo } \\
\hline \multicolumn{1}{|c|}{ Factor de riesgo } & \multicolumn{2}{c|}{ Sexo } & \multicolumn{2}{c|}{ Grupo Etario } \\
\hline & Masculino & Femenino & $\mathbf{2 0 - 3 9}$ & $\mathbf{4 0}-\mathbf{5 9}$ & $\mathbf{6 0}-\mathbf{7 5}$ \\
\hline Presión arterial elevada & $3.125 \%$ & $2.5 \%$ & $0 \%$ & $3.75 \%$ & $1.875 \%$ \\
\hline Hipertensión arterial, estadio I & $1.875 \%$ & $3.75 \%$ & $2.5 \%$ & $1.875 \%$ & $1.25 \%$ \\
\hline Hipertensión arterial, estadio II & $1.25 \%$ & $2.5 \%$ & $0 \%$ & $1.875 \%$ & $1.875 \%$ \\
\hline Crisis hipertensiva & $0.625 \%$ & $0 \%$ & $0.625 \%$ & $0 \%$ & $0 \%$ \\
\hline Glucemia elevada & $\mathbf{2 0} \%$ & $\mathbf{2 5 . 6 2 5} \%$ & $16.875 \%$ & $16.25 \%$ & $12.5 \%$ \\
\hline Diabetes mellitus & $\mathbf{3 . 7 5} \%$ & $\mathbf{8 . 1 2 5} \%$ & $1.875 \%$ & $3.75 \%$ & $6.25 \%$ \\
\hline Obesidad & $11.875 \%$ & $22.5 \%$ & $9.375 \%$ & $16.875 \%$ & $8.125 \%$ \\
\hline Consumo de alcohol & $\mathbf{2 7 . 5} \%$ & $\mathbf{1 1 . 2 5} \%$ & $19.375 \%$ & $12.5 \%$ & $6.875 \%$ \\
\hline Uso de tabaco & $\mathbf{6 . 8 7 5} \%$ & $7.5 \%$ & $1.875 \%$ & $8.125 \%$ & $4.375 \%$ \\
\hline Hipercolesterolemia & $15.625 \%$ & $17.5 \%$ & $6.875 \%$ & $16.875 \%$ & $9.375 \%$ \\
\hline Hipertrigliceridemia & $15 \%$ & $11.875 \%$ & $5.625 \%$ & $14.375 \%$ & $6.875 \%$ \\
\hline Colesterol HDL disminuido & $\mathbf{1 8 . 7 5 \%}$ & $\mathbf{2 0 . 6 2 5} \%$ & $\mathbf{1 8 . 7 5} \%$ & $14.375 \%$ & $6.25 \%$ \\
\hline Síndrome metabólico & $9.375 \%$ & $17.5 \%$ & $5.625 \%$ & $13.75 \%$ & $7.5 \%$ \\
\hline
\end{tabular}

Fuente: elaborado por Jiménez, Martínez, Reyes y Romano, noviembre - diciembre 2019.

En esta tabla, se presenta el porcentaje de la población estudiada que evidencia los factores de riesgo cardiovascular evaluados. Las elevaciones de glucemia fueron más frecuentes en el sexo femenino, en un 25.6 $\%$ (equivalente a 41 casos), que, en el sexo masculino, con un $20 \%$ (equivalente a 32 casos); la presencia de diabetes mellitus fue más alta en pacientes femeninas, en un $8.1 \%$ (equivalente a 13 casos), y en un $3.75 \%$ (equivalente a 6 casos) en pacientes masculinos. El consumo de alcohol fue mayor en pacientes masculinos, $27.5 \%$ (equivalente a 44 casos), en las mujeres, un $11.25 \%$ (equivalente a 18 casos); el uso del tabaco, la hipercolesterolemia y los niveles reducidos de colesterol HDL fueron los más alarmantes, debido a estar presentes en mayor porcentaje y distribución en los grupos etarios estudiados.

\section{Discusiones y conclusiones}

El riesgo cardiovascular a 10 ańos que más se presentó fue un RCV bajo, correspondiendo al $36.26 \%$ de la totalidad de casos que aplicaron para este riesgo (figura 1). Según un estudio realizado durante el periodo 2013-2014, titulado "Determinación de riesgo cardiovascular y edad vascular según el score de Framingham en pacientes del Hospital Nacional Arzobispo Loayza”, se encontró que el mayor porcentaje de la población de estudio presentó un 
bajo riesgo, con un $48.3 \%$ de los casos estudiados, lo cual concuerda con el resultado que se obtuvo en esta investigación 6 .

La American Heart Association (AHA), en su investigación "Prediction of Lifetime Risk for Cardiovascular Disease by Risk Factor Burden at 50 years of Age" expone sobre el efecto de los factores de riesgo cardiovascular en el riesgo cardiovascular de por vida, a 10 años y óptimo. El riesgo cardiovascular de por vida de la población de la presente investigación fue de $47.87 \%$ en hombres y $35.5 \%$ en mujeres, mientras que la AHA en su investigación halló un $51.7 \%$ en hombres y $39.2 \%$ en mujeres, se observa aproximación en los rangos encontrados entre esta investigación y el análisis de la AHA (véase tabla 1$)^{7}$.

El efecto individual de cada uno de los factores de riesgo cardiovascular no fue evaluado como tal, sino más bien la presencia de estos en la comunidad de Santana. La AHA, en el artículo anteriormente mencionado, expone sobre la asociación de las elevaciones de presión arterial y niveles de colesterol total elevados con un mayor riesgo cardiovascular de por vida; dichos factores estuvieron presentes en la población de estudio en un $28.1 \%$ para elevación de la presión arterial y en un $56.3 \%$ en el caso de dislipidemia, al cual corresponden un $33.1 \%$ por hipercolesterolemia (véase figura 2$)^{8}$.

La diabetes mellitus estuvo presente en un $13.10 \%$ y la hiperglucemia en un $65.6 \%$ en la población de estudio, es la que más se relaciona con mayores niveles de riesgo cardiovascular según la AHA, la cual presenta una asociación de los factores mencionados a una media de riesgo global de $67.1 \%$ en hombres diabéticos y un $57.3 \%$ en mujeres diabéticas. (véanse tabla 1 y figura 2 ).

La obesidad fue asociada a incrementos sustanciales del riesgo global y de 10 ańos, dicho factor estuvo presente en un $33.1 \%$ de la población estudiada. La presencia del tabaquismo en la población de estudio fue de un $13.80 \%$; el riesgo cardiovascular global y de 10 años calculados fueron significativamente más altos en aquellos pacientes que fumaban en contraste con los que no fumaban, sin embargo, el riesgo cardiovascular global obtenido de la AHA de los pacientes con o sin el factor de riesgo tabaquismo fue similar, aunque se registraron más eventos cardiovasculares tempranos en fumadores que en no fumadores en el análisis en cuestión (véase figura 2) 9 .

La revista Española de Cardiología, publicó "Metabolic Syndrome as a Cardiovascular Risk Factor in Patients With Type 2 Diabetes" una correlación positiva muy alta $\left(\mathrm{R}^{2}=5.5\right)$, dando a relucir una relación de: por cada criterio diagnóstico de síndrome metabólico, el riesgo cardiovascular del paciente aumenta $5.5 \%$; esto no se relaciona con los hallazgos encontrados de la investigación en la cual la correlación fue negativa $\left(\mathrm{R}^{2}=0.037\right)$. También difiere de los resultados hallados por la AHA en "Metabolic Syndrome as a Precursor of Cardiovascular Disease and Type 2 Diabetes Mellitus" en la cual se encontró una correlación positiva alta $\left(\mathrm{R}^{2}=1.54\right)$, pudiendo asociar el riesgo cardiovascular elevado al síndrome metabólico $^{10,11}$. Esta discrepancia entre los dos estudios puede ser por dos razones: la población de estudio tiene una distribución de riesgo cardiovascular a 10 años, bajo, en mayor proporción que el intermedio, o alto, y que muchos presentaron solo dos o tres criterios diagnósticos de síndrome metabólico, no tomándolos en cuenta de forma individual para la interpretación de la gráfica.

Según la AHA, en el artículoya mencionado, las elevaciones de glucemia, el consumo de alcohol, el uso de tabaco y la diabetes mellitus fueron de los factores de riesgo más prevalentes en su cohorte, asemejándose a los resultados obtenidos. Sin embargo, la distribución por sexo y grupo etario difieren de entre las dos investigaciones, los niveles de glucemia elevados tuvieron una distribución de un $40 \%$ en hombres y $44 \%$ en mujeres, mientras que en nuestra investigación fueron de un $20 \%$ hombres y un $25.6 \%$ mujeres, respectivamente. 
El uso de tabaco fue más frecuente en el sexo femenino con un $7.5 \%$, en contraste con los hombres en un $6.8 \%$, aunque la distancia entre ambos sea mínima, se correlaciona con los datos de la Revista de Cardiología Española sobre síndrome metabólico y factores de riesgo cardiovascular, donde el $47.1 \%$ de los fumadores eran del sexo masculino y $37.8 \%$ del sexo femenino. En esta investigación, el hecho de que los pacientes de sexo femenino representen un porcentaje mayor de uso de tabaco concuerda con el hecho de que las pacientes femeninas han comenzado a ser más prevalentes en el uso de este a nivel mundial ${ }^{12}$.

No se observó relación entre la medida de disminución de colesterol HDL por parte de la investigación realizada y los antecedentes encontrados, tomando en cuenta que la disminución de este de por sí es un factor de riesgo aparte de que el paciente padezca dislipidemia. Durante la recolección de datos, el desconocimiento de los pacientes sobre sus propios factores de riesgo y el hecho de que muchos se negaban a la toma de muestra fueron los principales factores limitantes de la investigación.

\section{Recomendaciones}

La OMS aconseja que los pacientes con enfermedades cardiovasculares o que tienen un alto riesgo cardiovascular cuenten con una detección temprana, con consejería y con apoyo farmacológico, tomando en cuenta que factores como la dislipidemia, obesidad, HTA y DM pueden contribuir al avance de su enfermedad si la misma ya está instaurada ${ }^{1}$. La AHA señala que en Estados Unidos solamente el $13 \%$ de una muestra nacionalmente representativa identificó las enfermedades cardiovasculares como la principal causa de muerte a nivel mundial, por lo que se considera un déficit sustancial del reconocimiento de las enfermedades cardiovasculares y el mismo riesgo cardiovascular ${ }^{10}$.

La OMS, OPS, AHA y National Heart, Lung, Blood Institute (NHLBI) afirman que la mejor herramienta para la prevención de las enfermedades cardiovasculares y el manejo del riesgo cardiovascular radica en la atención y prevención primaria. El personal de la salud debe enfocar su esfuerzo en estimar el riesgo cardiovascular de por vida de forma que las decisiones terapéuticas no estén basadas en un solo factor de riesgo, sino un manejo complementario de la situación actual del paciente. El riesgo cardiovascular a 10 ańos no debe ser el único en tomarse en cuenta, debido a que la estimación a corto plazo de los factores de riesgo se ha asociado en pacientes jóvenes a una poca adherencia, o incluso no iniciación a modificaciones del estilo de vida y tratamiento. El objetivo de la realización de esta investigación se origina en el reforzamiento de la salud cardiovascular; esto se basa en aplicar cambios en las políticas de salud del país, especialmente mediante el prisma de la prevención primaria dirigida a los puntos más débiles de la sociedad; personas con bajo nivel educativo y que viven en zonas rurales, ${ }^{9}$.

El National Cholesterol Education Program Expert Panel on Detection, Evaluation, and Treatment of High Blood Cholesterol in Adults (NCE-ATP III) exhorta considerar el riesgo cardiovascular de por vida en la práctica clínica de prevención primaria, especialmente en países en vía de desarrollo de Latinoamérica y el Caribe, con el objetivo de reducir el $80 \%$ de muertes por enfermedades cardiovasculares en países en vía de desarrollo reportado por la OMS en el 2016 como parte de los objetivos globales de la salud de para el $2030^{10}$.

\section{Referencias bibliográficas}

1. Organización Mundial de la Salud. (2019). ¿Qué son las enfermedades cardiovasculares? [online] Available from https://www.who.int/cardiovascular_diseases/about_cvd/es/ [Accessed 13 Dec. 2019].

2. Vu TH, Lloyd-Jones DM, Liu K, Stamler J, Garside DB, Daviglus ML. Optimal Levels of All Major Cardiovascular Risk Factors in Younger 
Age and Functional Disability in Older Age: The Chicago Heart Association Detection Project in Industry 32-Year Follow-Up Health Survey. Circ Cardiovasc Qual Outcomes. $2016 \mathrm{Jul} ; 9(4): 355-63$. Doi: 10.1161/CIRCOUTCOMES.115.002519. Epub 2016 Jul 5. PMID: 27382089; PMCID: PMC4956507.

3. Vega Abascal J, Guimará Mosqueda M, \& Vega Abascal L. (2019). Riesgo cardiovascular, una herramienta útil para la prevención de las enfermedades cardiovasculares. [online] Scielo.sld.cu. Available from: http://scielo.sld.cu/scielo.php?scrip$\mathrm{t}=$ sci_arttext\&pid=S0864-21252011000100010 [Accessed 13 Dec. 2019].

4. Álvarez Cosmea A. (2019). Las tablas de riesgo cardiovascular: Una revisión crítica. [online] Scielo.isciii.es. Available at: http:// scielo.isciii.es/scielo.php?script=sci_arttext \&pid =S $1131-57682001000300002$ [Accessed 13 Dec. 2019].

5. Factores de riesgo cardiovascular | Texas Heart Institute [Internet]. Texas Heart Institute. 2019 [cited 15 December 2019]. Available from: https://www.texasheart.org/heart-health/heartinformation-center/topics/factores-de-riesgo-cardiovascular/

6. Pichardo R, González Á, Ramírez W, Escaño F, Rodríguez C, Jiménez R. Estudio de los factores de riesgo cardiovascular y síndrome metabólico en la República Dominicana (EFRICARD II). Santo Domingo; 2019 p. 14.

7. Tormo M, Navarro C, Chiriaque M, Pérez D. Factores de riesgo cardiovascular en la región de Murcia, España [Internet]. España; 2017. Available from: http://scielo.isciii.es/ scielo.php?script=sci_arttext $\&$ pid $=S 11355$ 7271997000600002
8. The Facts About High\&nbsp;Blood Pressure [Internet]. www.heart.org. 2019 [cited 16 December 2019]. Available from: https://www. heart.org/en/health-topics/high-blood-pressure/ the-facts-about-high-blood-pressure

9. Cardiovascular risks of hypertension [Internet]. Uptodate.com. 2019 [cited 16 December 2019]. Available from: https://www.uptodate. $\mathrm{com} /$ contents/cardiovascular-risks-of-hypertension\#H1

10. Diagnosis and Classification of Diabetes Mellitus [Internet]. American Diabetes Association (ADA). 2019 [cited 16 December 2019]. Available from: https://care.diabetesjournals. org/content/37/Supplement_1/S81 diabetes

11. Tormo M, Navarro C, Chiriaque M, Pérez D. Factores de riesgo cardiovascular en la región de Murcia, España [Internet]. España; 2017. Available from: http://scielo.isciii.es/scielo.php?scrip$\mathrm{t}=$ sci_arttext\&pid=S1135-57271997000600002

12. Mayta J, Morales A, Cardenas A. Determinación de riesgo cardiovascular y edad vascular según el score de Framingham en pacientes del Hospital Nacional Arzobispo Loayza [Internet]. 2015 [cited 18 December 2019]. Available from: http://www.scielo.org.pe/pdf/hm/v15n2/ a05v15n2.pdf 
Anexo I. Formulario de recolección de datos

Formulario de recolección de datos:

ERCAS

Internado de Medicina Social - Santana, Peravia

Historia clínica $\quad$ Fecha:

Nombre:

Edad:

Sexo:

Código:

Nacionalidad:

Historia de la enfermedad actual:

Actual

Abandono

Nunca

Alcohol

$\square$ Drogas

$\checkmark$ Otros

D. Antecedentes familiares

- Padre

A. Vitales del paciente:

FC:

Madre

FR:

Hermanos/as

Otros parientes

TA:

SatO2:_ $\%$

$\mathrm{T}$ :

Glicemia:

E. Medidas del px:

Talla:

Peso:

IMC:

B. Antecedentes personales (si el paciente confirma, especificar cual, duración y el tx):

Cardiovascular

- Endocrinológico

๑ Gastrointestinal

․ Genitourinario

$\square$ Respiratorio

Neuro-muscular

$\square$ Hematológico

$\square$ Quirúrgico

(- Trauma

Ginecológico (cuestionar sobre trastornos hipertensivos del embarazo)

C. Hábitos tóxicos

F. Farmacología (¿̨cuáles fármacos toma actualmente?), especificar:

Antihipertensivos

- Hipoglucemiantes

- Anticoagulantes

$\square$ Diuréticos

Otros

G. Niveles de colesterol total, HDL, LDL, triglicéridos.

- C-TOTAL

- HDL

- TGL

- C-LDL

Cigarrillos 
Evaluación del riesgo cardiovascular en adultos del distrito municipal de Santana, Peravia, República Dominicana, durante el periodo noviembre - diciembre 2019 (ERCAS I)

Anexo II. Consentimiento informado, formalizado para todas las investigaciones del Internado de Medicina Social.

\section{Consentimiento informado para la publicación de información personal}

A través de este medio doy mi consentimiento para que todo el material de la historia clínica, imágenes y cualquier otro tipo de información acerca del paciente mencionado a continuación, sea publicado en una revista médica o congreso médico que los autores consideren pertinentes con fines científicos y docentes.

Nombre del paciente:

Comprendo que no se publicará mi nombre o el nombre de mi familiar y que se intentará en todo lo posible mantener el anonimato de la identidad en el texto y en las imágenes. Sin embargo, comprendo que no se puede garantizar el anonimato completo.

Esta autorización incluye la publicación en idioma español y su traducción al inglés, impresa, en formato electrónico en el sitio web de la revista, y en cualquier otro formato usado por la revista cientifica actualmente y en el futuro.

La revista está destinada a los médicos pero puede ser leída por otras personas que no son médicos.

Puedo revocar mi consentimiento en cualquier momento antes de la publicación, pero una vez que la información haya sido consignada para publicación ("en imprenta") ya no será posible revocar el consentimiento.

A través de este medio manifiesto a la persona o institución correspondiente que he entendido y aprobado lo mencionado con anterioridad.

Nombre del padre, madre o tutor legal:

Documento de identidad (tipo y número): Parentesco:

Firma: Fecha:

Firma y sello del profesional. 